\title{
Aquilegia saxifraga sp. nov. (Ranunculaceae) en la provincia de Málaga (España)
}

\author{
Federico Casimiro-Soriguer Solanas \& Baltasar Cabezudo \\ Área de Botánica (Departamento de Biología Vegetal). Facultad de Ciencias. Universidad de Málaga. Málaga. *Autor \\ para correspondencia: fedeque@ hotmail.com
}

\section{Correspondencia}

F. Casimiro-Soriguer Solanas

e-mail: fedeque@hotmail.com

Recibido: 11 mayo 2018

Aceptado: 20 julio 2018

Publicado on-line: septiembre 2018
Aquilegia saxifraga sp. nov. (Ranunculaceae) from Málaga province (Spain)

Palabras clave: Aquilegia, flora, endemismo, Málaga, ZEC de Camarolos, Andalucía.

Key words: Aquilegia, flora, endemism, Málaga, ZEC Camarolos, Andalusia
El género Aquilegia L. (Ranunculaceae), con aproximadamente 70 especies descritas (Munz, 1946; Nold, 2003), tiene una amplia distribución en el hemisferio Norte y se reparte por las zonas templadas de Asia, Europa, Norteamérica, Macaronesia y Norte de África. Díaz (1986) reconoce para la Península Ibérica 3 especies: $A$. vulgaris L., A. pyrenaica DC. y A. viscosa Gouan, que agrupan a 10 taxones en total. Posteriormente a la síntesis de Díaz (1986) no se ha descrito ningún nuevo taxón del género en la Península Ibérica, aunque sí se ha reivindicado (Montserrat \& Montserrat, 1988) el status específico para $A$. pyrenaica subsp. guarensis (Losa) Rivas-Mart. como $A$. aragonensis Willk. y el de $A$. paui Font Quer (A. vulgaris subsp. paui (Font Quer) O. Bolos \& Vigo) (Martinell et al., 2007).

Según Díaz (1986), el género Aquilegia está representado en Andalucía solo en su mitad oriental y por cuatro taxones: $A$. vulgaris $\mathrm{L}$. subsp. vulgaris, A. vulgaris subsp. nevadensis Boiss. \& Reut., $A$. vulgaris subsp. hispanica (Willk.) Heywood y $A$. pyrenaica subsp. cazorlensis (Heywood) Galiano \& Rivas Martínez, todas ellas vivaces, con cepas leñosas y diferenciadas fundamentalmente por el tamaño de la planta, la morfología de los espolones florales y de los estaminodios. Ecológicamente las subespecies de $A$. vulgaris viven en bosques, prados y lugares húmedos, mientras que $A$. pyrenaica subsp. cazorlensis lo hace sobre roquedos calizos umbríos. En la Flora Vascular de Andalucía Oriental (Morales Torres \& Vizoso, 2009) el género Aquilegia se localiza exclusivamente en las sierras Béticas Orientales y está representado por tres taxones: $A$. vulgaris subsp. nevadensis, A. vulgaris subsp. hispanica, ambas ligadas a pastizales y herbazales en zonas húmedas y A. pyrenaica subsp. cazorlensis ligada a un hábitat subrupícola en umbrías.

El género Aquilegia está siendo objeto en los últimos años de numerosos trabajos sobre evolución y filogenia (Bastida et al., 2010; Fior et al., 2013), en los cuales se indica que las poblaciones del género en el sur de Europa están en pleno proceso de diversificación, motivado fundamentalmente por el aislamiento geográfico y la especialización al hábitat. En uno de estos trabajos (Garrido et al., 2017) sobre distribución geográfica y genética realizado en la Península Ibérica, los autores distinguen 5 linajes puros asignados a A.vulgaris subsp. vulgaris (en la que incluyen a A.vulgaris subsp. hispanica), A.vulgaris subsp. nevadensis, $A$. vulgaris subsp. dichroa (Freyn) T.E. Díaz, A. pyrenaica subsp. cazorlensis y $A$. pyrenaica subsp. pyrenaica.

Durante las campañas de herborizaciones llevadas a cabo para la elaboración de la flora y vegetación de la ZEC Sierra de Camarolos (Cabezudo et al., 2016), localizamos una pequeña población del género Aquilegia con unas características morfológicas, biológicas y ecológicas que no se correspondían globalmente con ninguna de las especies descritas para Andalucía y que consideramos como una especie independiente.

Aquilegia saxifraga Casimiro-Soriguer Solanas \& Cabezudo, sp. nov.

Difiere del complejo de Aquilegia vulgaris L. de la Península Ibérica por su menor tamaño, inflorescencias menores y cepa aérea, leñosa, 


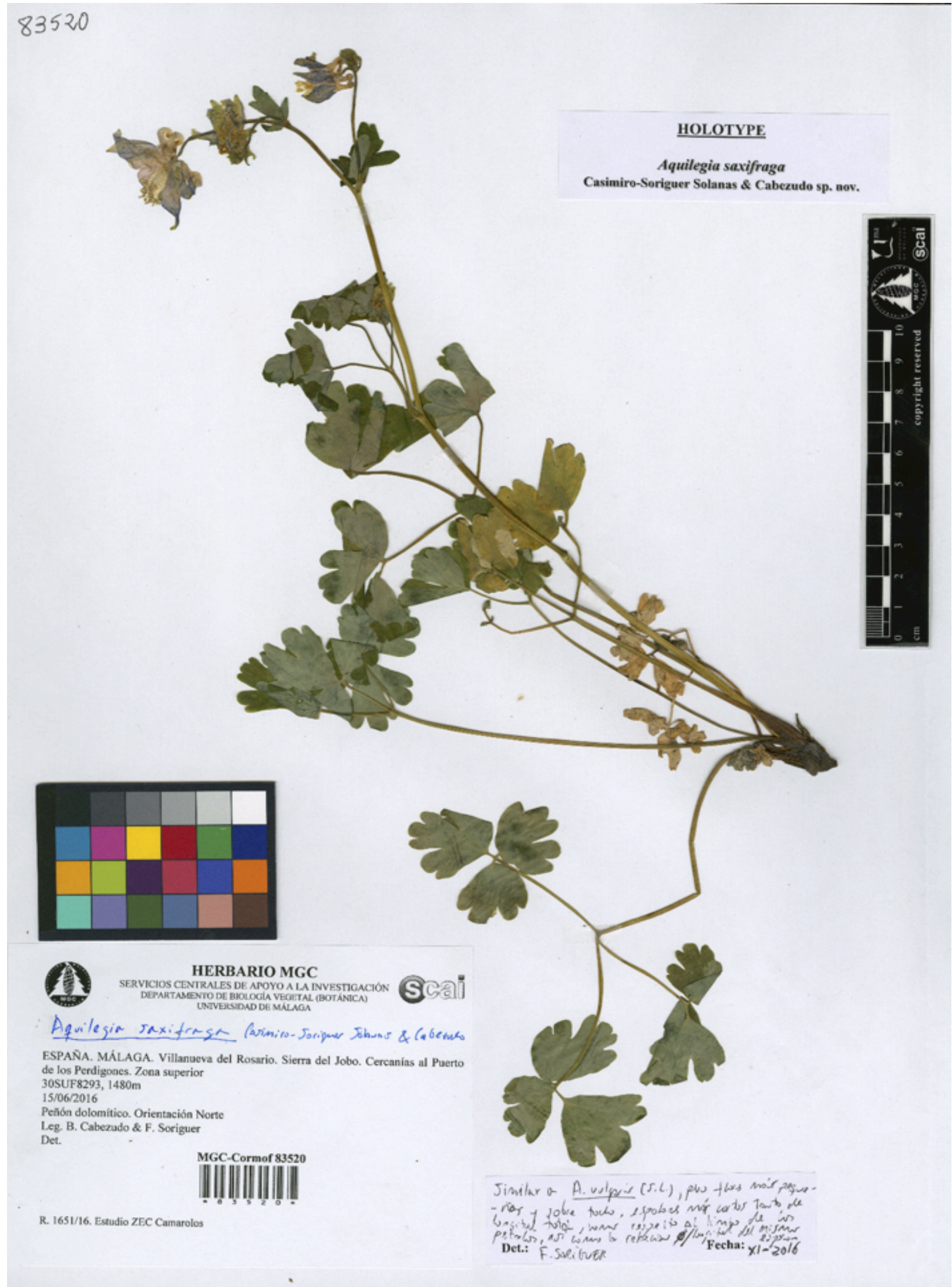

Figura 1. Holotypus de Aquilegia saxifraga sp. nov. (MGC 83520)

Figure 1. Holotype of Aquilegia saxifraga sp.nov 
alargada y cubierta de las vainas de las hojas viejas de años anteriores. De A. pyrenaica DC. (s.I.) por su cepa aérea, sus flores con estaminodios oblongos, espolones marcadamente uncinados y hojas con folíolos mayores. De A. viscosa subsp. hirsutissima (Timb.-Lagr.) Breistr., por su cepa aérea, sus flores con estaminodios oblongos y estambres exertos y tallo no glanduloso-viscoso. De A. paui Font Quer., por su cepa aérea, mayor porte, flores más grandes con espolones más largos y hojas biternadas.

Differs from Iberian Aquilegia vulgaris L. complex by being smaller, inflorescence smaller too, and in having aerial stock stout, with abundant shuck from old leaves of old years. From $A$. pyrenaica DC. (s.I.), in having aerial stock stout, flowers with oblong staminodes and strong curved spur, leaves with bigger leaflets. From $A$. viscosa subsp. hirsutissima (Timb.-Lagr.) Breistr., in having aerial stock stout, flowers with oblong staminodes and exert stamens, and stem not viscid. From $A$. paui Font Quer in having aerial stock stout, by being taller, in having bigger flowers with bigger spur and biternate leaves.

Holotypus: España. Málaga. Villanueva del Rosario. Sierra del Jobo. 1480 m.s.m. 15/06/2016. Peñón dolomítico, orientación norte. B. Cabezudo \& F. Soriguer. MGC 83520. (Figura 1).

Paratypus: España. Málaga. Villanueva del Rosario. Sierra del Jobo. 1480 m.s.m. 14/06/2017. Comunidades rupícolas dolomitícolas. F. Soriguer \& J. García-Sánchez MGC 86606.

Etimología: saxifraga, del latín saxum ("piedra") y frangere (“romper, quebrar").

Descripción. Casmófito perenne, camefítico. Cepa aérea, leñosa, alargada, más o menos tortuosa, cubierta de las vainas de las hojas viejas de años anteriores (Figura 2d). Rizoma delgado. Escapo hasta $30 \mathrm{~cm}$ y de hasta $2 \mathrm{~mm}$ de grosor, escasamente ramificado en la parte superior, glabro solo en su parte media, pubescente hacia la parte distal, con indumento similar al de la inflorescencia, laxamente pubescente en su parte basal con pelos largos no glandulíferos. Roseta basal con 2 a 5 hojas, con pecíolos pubescentes no glandulares, de más de $20 \mathrm{~cm}$, biternadas. Folíolos redondeados de 2-3 ' $2-2,5 \mathrm{~cm}$, profundamente lobulados, con los lóbulos redondeados, de sésiles a cortamente peciolulados, de haz verde y glabro y envés glauco con pubescencia de pelos largos no glandulíferos. Hojas inferiores del escapo 1 o 2, de morfología similar a las de la cepa, aunque menores, e indumento como las inferiores, tanto en distribución como en morfología. Hojas asociadas a la inflorescencia $3-4$, menores que las inferiores, de cortamente pecioladas a sésiles, una vez ternadas o con un solo folíolo profundamente dividido, pubescencia como las inferiores, tanto en distribución como en morfología. Inflorescencia de hasta $12 \mathrm{~cm}$ (medida desde la intersección de la rama de la flor más inferior hasta la última flor del escapo), con 4-8 flores. Ramas de la inflorescencia densamente glandular-pubescentes con pelos largos y cortos glandulíferos y glándulas sentadas. Flores con pedúnculos glandular-pubescentes, nutantes, azules, concoloras, hasta de $3,5 \mathrm{~cm}$ de diámetro. Sépalos de 21-30 x 5-9 mm, lanceolados, erectos, acuminados, con ápice verdoso-blanquecino, pubérulos en la parte externa y con pelos cortos glandulíferos en los márgenes. Pétalos de 21-23 $\mathrm{mm}$ de longitud (limbo más espolón), con pelos glandulíferos; limbo de 7-8,5 x 10 mm, espolón de 15-16 × $2 \mathrm{~mm}$, marcadamente uncinado (Figura 3a). Estambres netamente exertos, numerosos, los internos transformados en estaminodios oblongolanceolados, de márgenes ondulados y apiculados en su parte distal, de aproximadamente $7 \times 2 \mathrm{~mm}$. Carpelos tantos como folículos. Folículos 5(6), de $14 \mathrm{~mm}$ de longitud (en el estadío observado), densamente glandular-pubescentes, con pelos cortos glandulíferos y glándulas sentadas; estilos persistentes, ligeramente glandulosos. Semillas no estudiadas.

Distribución. Endemismo estricto de la provincia de Málaga, localizado en la zona cacuminal de la Sierra del Jobo (provincia Bética, sector Antequerano, subsector Torcalense, cf. Cabezudo et al., 2016), perteneciente al término municipal de Villanueva del Rosario (Málaga, Andalucía, España) (Figura 4).

Hábitat. Rupícola. En pequeñas oquedades y fisuras de paredes verticales de rocas dolomíticas con exposición norte y muy umbrías, de 1450-1500 m., piso supramediterráneo y ombroclima húmedo. En el mismo hábitat se encuentran (cf. Cabezudo et al., 2016) Asplenium ruta-muraria L. subsp. ruta-muraria, A. trichomanes subsp. quadrivalens D.E. Meyer, Rhamnus pumilus Turra, Campanula rotundifolia subsp. hispanica (Willk.) O. Bolós \& Vigo, Hieracium amplexicaule L., $H$. texedense Pau, H. baeticum Arv.-Touv. \& É. Rev., Erinus alpinus L. y Arabis serpillifolia Vill., todas ellas en una comunidad perteneciente al Saxifragion camposii (Potentilletalia caulescentis, Asplenietea trichomanis) (Figura 3b). 
Fenología. Florece desde mediados de junio hasta principios de Julio, mes en el que comienza a fructificar.

Demografía y reproducción. La única población conocida hasta el momento ocupa una superficie aproximada de $100 \mathrm{~m}^{2}$ y está compuesta por 17 ejemplares. En el año 2016 solo uno de ellos estaba en flor (recolectada la parte aérea para el ejemplar tipo) y en el año 2017 florecieron dos individuos de los que recolectamos exclusivamente algunas flores (parátipo). Se ha observado polinización por Bombus sp. Hemos observados un buen desarrollo inicial de los folículos, pero no hemos hecho un seguimiento de la maduración de los mismos.

Amenazas. Consideramos, en base a la pequeña superficie que ocupa y al bajo número de ejemplares, que la especie se encuentra En Peligro Crítico (CR) según los criterios establecidos por UICN (2012): B2ab(i, ii); D1+2. Según la legislación ambiental de España, es una especie En Peligro (EN) y merecedora de ser protegida por la legislación de Andalucía.

Observaciones. Las características del aparato vegetativo (tipo biológico), cepa y rizoma de los taxones ibéricos del género Aquilegia, no están claramente descritas en la bibliografía utilizada, pero están bien representadas en la iconografía de dicha bibliografía (Díaz, 1986; Villar et al., 1997-2001) y en el material de herbario de todas las especies ibéricas que hemos estudiado. En base a lo anterior hemos observado la existencia de dos grupos de especies en la Península Ibérica. Por un lado, estarían $A$. viscosa, $A$. paui y $A$. pyrenaica (s.l.) en las que el aparato vegetativo consta de una cepa leñosa, no aérea, alargada y cubierta por las vainas de las hojas viejas de años anteriores, carácter ya indicado para A. pyrenaica (Rivas Martínez, 1967), y que se continua con un rizoma generalmente más fino que la cepa. Por otro lado se encuentran los taxones incluidos por Díaz $(1984,1986)$ en $A$. vulgaris s.l. (excepto A.vulgaris subsp. paui), que presentan una cepa corta, no aérea, sin restos o escasos de hojas viejas de años anteriores y que se continua por un grueso rizoma de anchura similar a la cepa. Excepto Morales Torres \& Vizoso (2009), que consideran a las especies andaluzas del género como geófitos rizomatosos, el resto de bibliografía consultada donde se detalla el tipo biológico de los distintos taxones ibéricos del género Aquilegia considera a estos como hemicriptófitos (Villar et al., 1997-2001; Herrera et al., 1998; Sáez Goñalons
\& Guardia Valle, 2004), criterio que compartimos en base a nuestras observaciones. A. saxifraga tiene la cepa con una morfología similar a la del grupo de A. pyrenaica, A. paui y A. viscosa, pero se diferencia de este grupo por sus cepas aéreas, comportándose como un caméfito y no como un hemicriptófito (Figura 2). El aislamiento geográfico, sus características vegetativas y el hábitat de esta nueva especie se adaptan a la especiación alopátrica del género en la Península Ibérica, ligada a medios rocosos y fríos, y que afecta sobre todo a caracteres vegetativos (Medrano et al., 2006; Alcántara et al., 2010; Jaime et al., 2015).

La población de $A$. saxifraga es la más suroccidental del género en la Península Ibérica (Jaime et al., 2015; Morales Torres \& Vizoso, 2009), estando totalmente aislada del resto de poblaciones del género Aquilegia presentes en Andalucía, siendo las más cercanas las poblaciones de $A$. vulgaris subsp. nevadensis que se localizan a unos $25 \mathrm{Km}$ de distancia, en la vertiente norte de Sierra Tejeda (Málaga y Granada), ligadas estas últimas a suelos con humedad edáfica permanente (Cabezudo et al., 2005, 2006). A. saxifraga tiene un hábitat y una estructura poblacional acorde con otras especies endémicas del sur de Europa ligadas a medios rocosos y con escasos individuos como A. paui, especie endémica de Tarragona, glerícola, con un área de $1 \mathrm{~km}^{2}$ y menos de 300 individuos (Martinell et al., 2007) y $A$. nurargica Arrigoni \& E. Nardi, endemismo de Cerdeña, rupícola y con unos 40 individuos (Fenu et al., 2011).

\section{Materiaal estudiado}

Aquilegia paui Font Quer

Tarragona: Tossa de Caro (MA 3889; BC 815955).

Aquilegia pyrenaica subsp. cazorlensis (Heywood) Galiano \& Rivas Martínez

Jaén: Sierra de Cazorla (GDA 35155; MA 439791; MA 350069; MA 457744;; MA 208111; MA 350068; MA 196633; GDA 5415; GDA 998); Sierra del Pozo (GDA 9714); Quesada (MA 457743; MA 482616); Pozo Alcón (MA 457742; MA 482626; MA 482629; MA 482628, MA 457744, MA 208111); Sierra de Segura (MA 233480).

Aquilegia pyrenaica subsp. discolor (Levier \& Leresche) Pereda \& Laínz

Asturias: Pico Valverde (FCO 35714); Picos de Europa (FCO12339; FCO12337; FCO12336; FCO12332, FCO12333; FCO12330). Cantabria: Picos de Europa (FCO12335; FCO12334). León: 


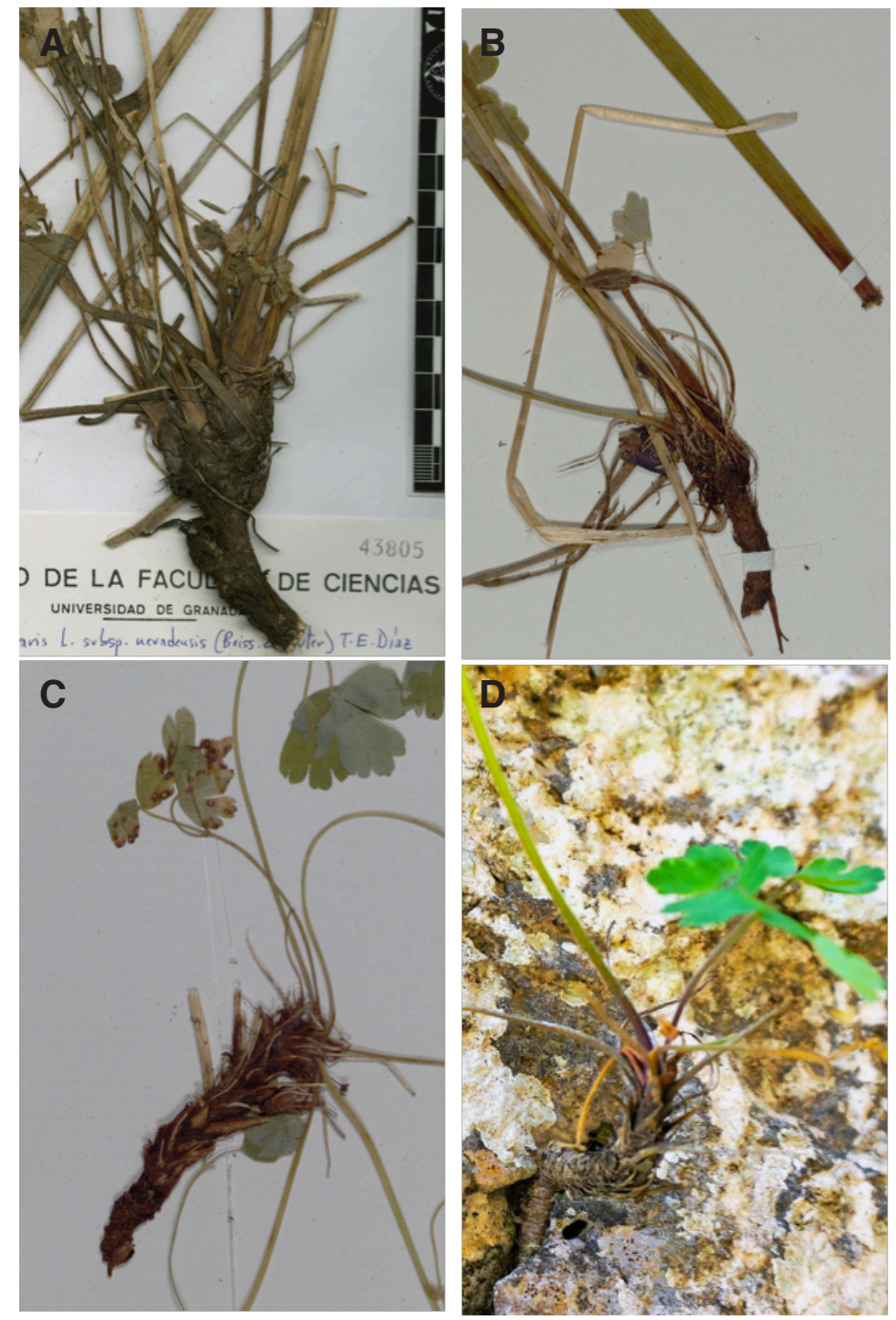

Figura 2. Parte basal (cepa y rizoma) de los 4 taxones andaluces de Aquilegia: A. Aquilegia vulgaris subsp. nevadensis (GDA 43805); B. Aquilegia vulgaris subsp. hispanica (MA 550965); C. Aquilegia pyrenaica subsp. cazorlensis (MA 457742); D. Aquilegia saxifraga sp. nov. (hábitat natural).

Figure 2. Basal part (rhizome and stockstout) from 4 Andalusian taxa of Aquilegia.

Picos de Europa (FCO12331).

Aquilegia pyrenaica subsp. guarensis (Losa) Rivas Martínez

Huesca: Sierra de Guara (VIT 91547; SEV 20338); Glera de Guara (BC 110667).

\section{Aquilegia pyrenaica DC. subsp. pyrenaica}

Navarra: Isaba (MGC 32491, MA 878464).

Cantabria: San Roque de Riomera (MA 691458).

Burgos: Espinosa de los Monteros (MA 472257); Valle de Mena (MA 468622). Álava: Amurrio (MA 338331); Arztz (MA 349877). Huesca: Pirineos (MGC 712); Ansó (MA617359); Borao (MA231191).
Pirineo Aragonés (MA 293516, MA 472257); Canfranc (MA 165829); Candanchú (MA 195145); Benasque (MA 231186). Lérida: Valle de Arán (MA 38911); Maladeta (MA 38910); Les Bordes (MA 490252). Vizcaya: Gorbea (MA 349876).

Aquilegia viscosa subsp. hirsutissima (Timb.Lagr.) Breistr.

Lérida: Alt Urgell (BCN 32919); Cadí (BCN 94159); Gisclareny (BCN 94160); Serra del Moixero (BCN 94164); Serra Pedregosa (BCN 94167).

Aquilegia vulgaris subsp. dichroa (Freyn) T.E. Díaz 

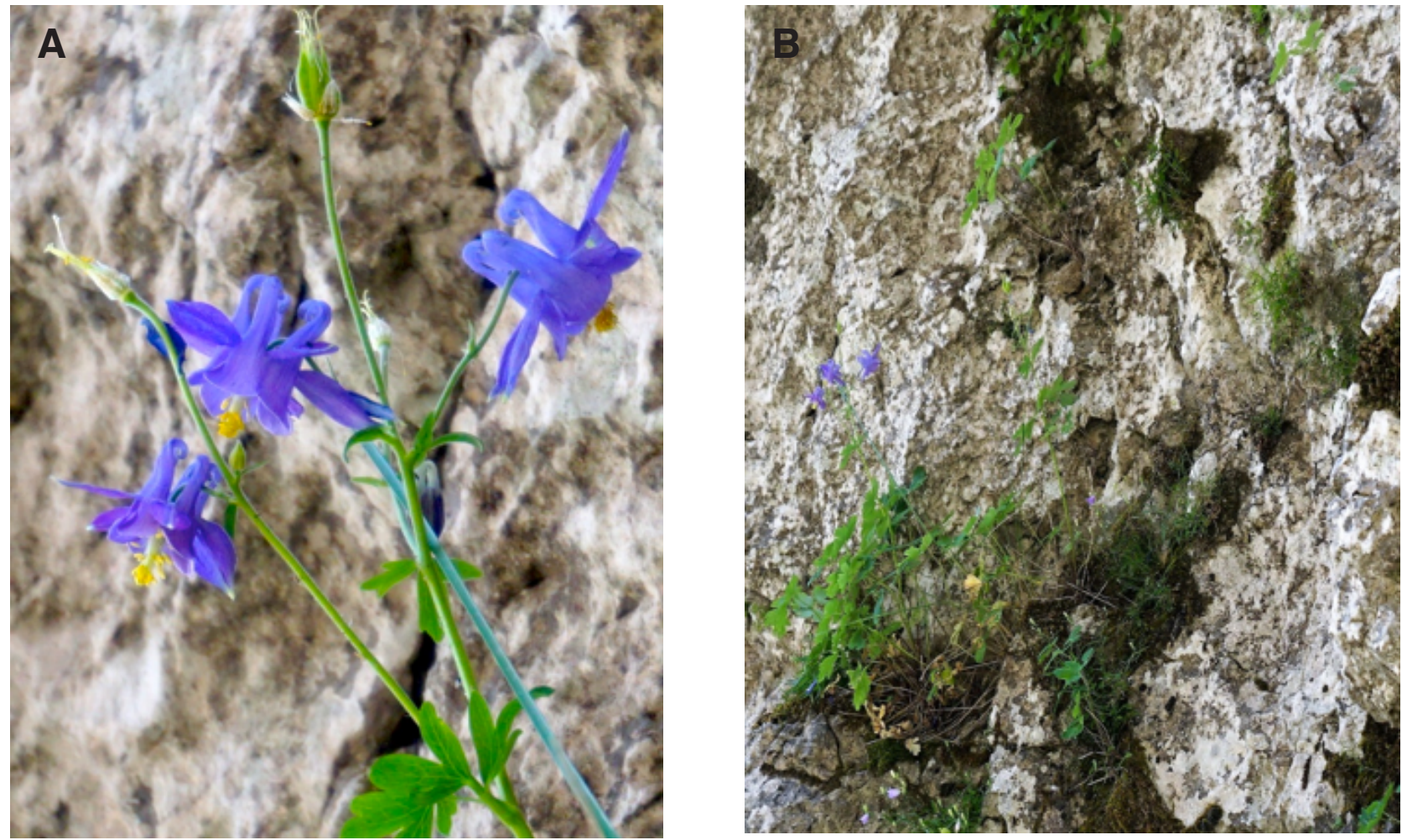

Figura 3. Aquilegia saxifraga sp. nov.: A. Inflorescencia; B. Hábitat.

Figure 3. A. Inflorescence; B. Habitat.

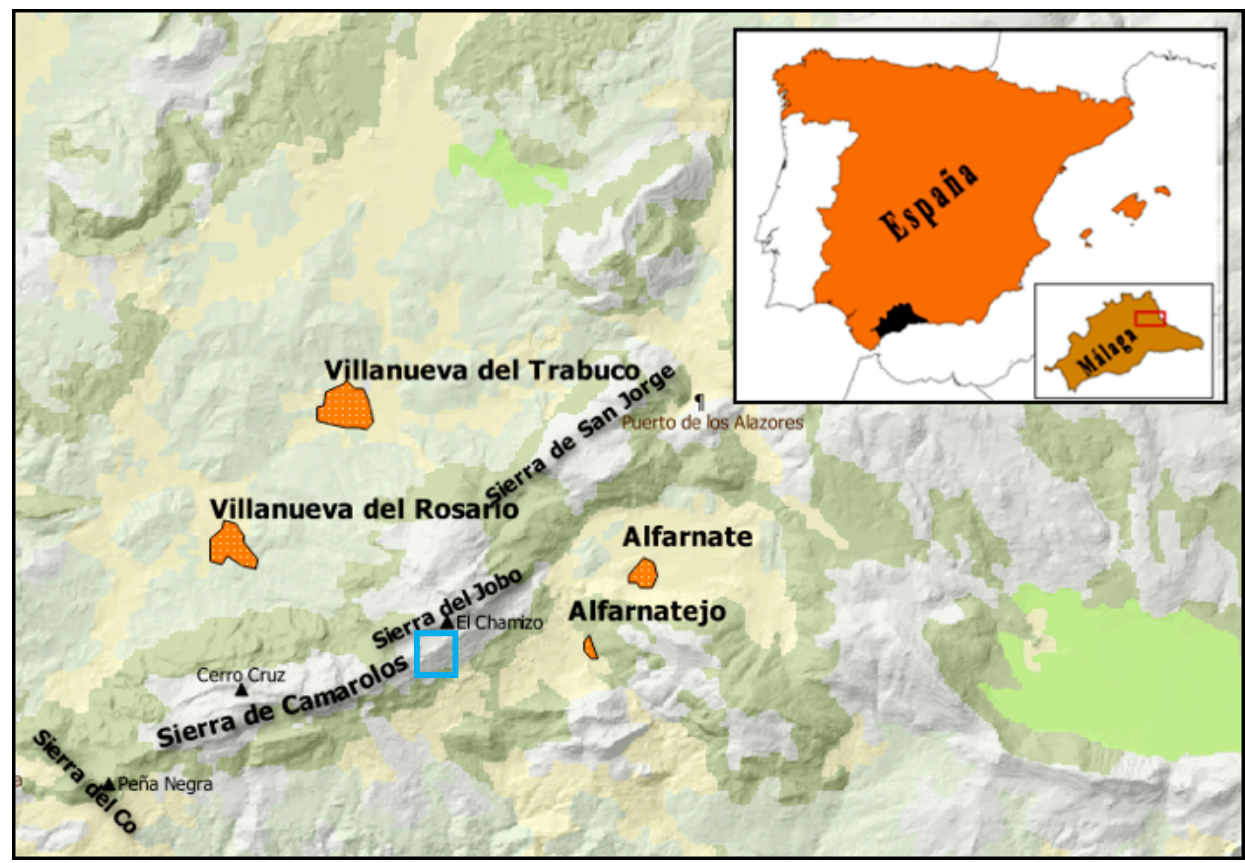

Figura 4. Situación geográfica de la ZEC Sierra de Camarolos (Málaga, España). Recuadro azul, localización de la única población conocida de Aquilegia saxifraga.

Figure 4. Location of the ZEC Sierra de Camarolos (Málaga, Spain). Blue square, location of only known population of Aquilegia saxifraga. 
Ávila: Pto. Miares (MA 257992). Cáceres: Garganta la Olla (SALA 15421); Robledillo (SALA 8577); Baños de Montemayor (MA 38880). León: Isoba (MA 777372); Ponferrada (SALA 42916, MA 316852); La Baña (MA 316853). Madrid: Pto. de Canencia (SALA 9158). Zamora: Galende, Ribadelago, Bouzas (SALA 133906); Justel (SALA 132070); Figueruela de Arriba (SALA 133885); Vega del Castillo (SALA 5359). Salamanca: Entre Navacarros y Candelario (SALA 20388); La Honfría (SALA 5174); La Alberca (MA 38878, MA 38876).

\section{Aquilegia vulgaris subsp. hispanica (Willk.) Heywood}

Granada: Sierra de Huétor (GDA 48689). JAÉN: Sierra de Segura (GDA 13114); Santiago de la Espada (MA 550965; MA 52261). Teruel: Sierra de Gúdar (MA170058-2); Sierra de Albarracín (MA 38931); Griegos (MA 38929). Guadalajara: Peñalén (MA 233478). Segovia: Rascafría (MA 233477-2). Cuenca: Hoz de Beteta (MA 38858). Madrid: Somosierra (MA 233479); Rozas de Pto. Real (MA 556902); Cercedilla (MA 231183); Montejo de la Sierra (MA 38855); Dehesa de Somosierra (MA 38856).

Aquilegia vulgaris subsp. nevadensis (Boiss. \& Reut.) T.E. Díaz

Almería: Pto. de La Ragua (GDA 15531). Granada: Barranco del río Válor (GDA 8029); Campos de Otero (GDA 35156); Sierra de Baza (GDA 15532; GDA15529); Sierra Nevada (GDA 15535; GDA43857; GDA43805; MA 439788; MA 394719); Sierra Tejeda, (GDA 15533; GDA 15534; MGC 6887; MGC 59764; MA 38841); MGC 54660); Río Barrio (GDA 2428; MGC 4818); Laroles (GDA 28765; MGC 18149); Dehesa de Camarate (MA 752835); Trevenque (MA 233476). Málaga: Sierra Tejeda (MGC 18150, MA 38882).

\section{Aquilegia vulgaris $\mathrm{L}$. subsp. vulgaris \\ Álava: Okina (VIT 2353; VIT 2354); Subijana (VIT 2358). Huesca: Selva de Oza (GDA 37821).}

\section{Agradecimientos}

Nuestro agradecimiento a los herbarios que nos han facilitado los pliegos utilizado en este trabajo. Igualmente agradecemos la colaboración de José García Sánchez y Andrés Vicente Pérez Latorre en las tareas de campo durante la elaboración de la flora de la ZEC Sierra de Camarolos.

\section{Bibliografía}

Alcántara, J.M., Bastida, J.M. \& Rey, P.J. (2010). Linking divergent selection on vegetative traits to environmental variation and phenotypic diversification in the Iberian columbines (Aquilegia). J. Evol. Biol., 23, 12-18.

Bastida, J.M., Alcántara, J.M., Rey, P.J., Vargas, P., Herrera, C.M. (2010). Extended phylogeny of Aquilegia: the biogeographical and ecological patterns of two simultaneous but contrasting radiations. PI. Syst. Evol., 284, 171-185.

Cabezudo, B., Pérez-Latorre, A.V., Navas, D., Gavira, O. \& Caballero, G. (2005). Contribución al conocimiento de la Flora del Parque Natural de las Sierras Tejeda, Almijara y Alhama (Málaga-Granada, España). Acta Bot. Malacitana, 30, 55-110.

Cabezudo, B. \& Pérez-Latorre, A.V. (2006). Datos sobre la vegetación relicta con Gymnocarpium robertianum (Hoffm.) Newman en el sur de la Península Ibérica. Acta Bot. Malacitana, 31, 174-177.

Cabezudo, B., Casimiro-Soriguer Solanas, F., GarcíaSánchez, J. \& Pérez-Latorre A.V. (2016). Flora y vegetación de la Zona de Especial Conservación (ZEC) Sierra de Camarolos (Málaga, España). Acta Bot. Malacitana, 41, 63-246.

Díaz, T.E. (1986). Aquilegia L. In S. Castroviejo et al. (Eds.), Flora iberica 1 (pp. 376-387). Madrid: Real Jardín Botánico.

Díaz, T.E. (1984). Nota sobre Aquilegia vulgaris L., s.l., en la Península Ibérica. Anales Jard. Bot. Madrid, 41(1), 210-211.

Fenu, G., Mattana, E., Congiu, A., Garrido, J.L. \& Bacchetta, G. (2011). Aquilegia nuragica Arrigoni \& E. Nardi. Inform. Bot. Italiano, 43 (2), 381-458.

Fior, S., Li, M.G., Oxelman, B., Viola, R., Hodges, S.A., Ometto, L. \& Varotto, C. (2013). Spatio temporal reconstruction of the Aquilegia rapid radiation through next-generation sequencing of rapidly evolving cpDNA regions. New Phytol., 198, 579-592.

Garrido, J.L., Alcántara, J.M., Rey, P.J., Medrano, M., Guitian, J., Castellanos, M.C., Bastida, J.M., Jaime, R. \& Herrera, C.M. (2017). Geographic genetic structure of Iberian columbines (gen. Aquilegia). Pl. Syst. Evol., 303, 1145-1160.

Herrera, C.M., Hernández-Bermejo, E., Luque, P. \& Benavente, A. (1998). Aquilegia pyrenaica subsp. cazorlensis. In G. Blanca, B. Cabezudo, E. HernándezBermejo, C.M. Herrera, J. Molero Mesa, J. Muñoz \& B. Valdés (Eds.), Libro Rojo de la Flora Silvestre de Andalucía, tomo 1: especies en peligro de Extinción (pp. 53-55). Sevilla: Consejería de Medio Ambiente, Junta de Andalucía.

Jaime, R., Alcántara, J.M., Bastida, J.M. \& Rey, P.J. (2015). Complex patterns of environmental niche evolution in Iberian columbines (genus Aquilegia). J. Pl. Ecol., 8(5), 457-467.

Martinell, M.C., Sáez, L. \& Molero, J. (2007). Taxonomic assessment of the critically endangered narrow endemic Aquilegia paui Font Quer. In: Llibre de resums del XII OPTIMA Meeting. Pisa, 12-16 de setembre de 2007.

Medrano, M., Castellanos, M.C. \& Herrera, C.M. (2006). Comparative floral and vegetative differentiation between two European Aquilegia taxa along a narrow contact zone. Pl. Syst. Evol., 262, 209-224. 
Montserrat Martí, G. \& Montserrat Martí, J. M. (1988). Aquilegia aragonensis Willk., endemismo pirenaico poco conocido. Lagascalia, 15 (extra), 177-180.

Morales Torres, C. \& Vizoso, M.T. (2009). Aquilegia L. In G. Blanca, B. Cabezudo, M. Cueto, C. Fernández López \& C. Morales Torres (Eds.), Flora vascular de Andalucía Oriental, Vol. 2 (pp. 46-47). Sevilla: Consejería de Medio Ambiente, Junta de Andalucía. Sevilla.

Munz, P.A. (1946). Aquilegia. Gentes Herb., 7, 1-150.

Nold, R. (2003). Columbines. Aquilegia, Paraquilegia and Semiaquilegia. Cambridge: Timber Press.

Rivas Martínez, S. (1967). Taxonomía del grupo Aquilegia pyrenaica DC. Bol. Real Soc. Española Hist.
Nat. (Biol.), 65, 107-109.

Sáez Goñalons, L. \& Guardia Valle, L. (2004). Aquilegia vulgaris subsp. paui. In A. Bañares, G. Blanca, J. Güemes, J.C. Moreno \& S. Ortiz (Eds.), Atlas y Libro Rojo de la Flora Vascular Amenazada de España (pp. 104-105). Madrid: Dirección General de Conservación de la Naturaleza.

UICN (2012). Categorías y criterios de la lista roja de la UICN: Versión 3.1. Segunda edición. Gland, Suiza y Cambridge: UICN.

Villar, L., Sesé, J. A. \& Ferrández, J.V. (1997/2001). Flora del Pirineo Aragonés vol. I. Huesca: Instituto de Estudios Altoaragoneses \& Consejo de Protección de la Naturaleza de Aragón. 